\title{
Etiological Pattern of Dementia in Bangladesh
}

\author{
KHAN MRK ${ }^{1}$, RIZVI AN ${ }^{1}$, HABIB MA $^{2}$, HASAN MK ${ }^{3}$, MAMUN $^{4}$, ALAM MR ${ }^{5}$, ISLAM R ${ }^{6}$
}

\begin{abstract}
:
Background: Dementia is a chronic \& progressive neurodegenerative disorder affecting usually older people of more than 65 years in which there are disturbances of multiple higher cortical functions including memory, thinking, orientation \& others. Dementia patients are increasing in number as the population of older age group is increasing. All types of dementia are treatable, at least with psychosocial interventions. So, accurate diagnosis and evaluation of etiological pattern is essential. Methods: This cross sectional study was carried out in the Department of Neurology, Bangabandu Sheikh Mujib Medical University, Dhaka, Bangladesh from January 2012 to December 2012 on 88 patients with dementia diagnosed on the basis of mini mental state examination and DSM-IV criteria. Results: Vascular dementia was the underlying diagnosis in most of the cases (43.3 \%) followed by Alzheimers Disease (20.2\%) and Parkinson Disease (9\%). Other causes were Mixed Dementia, Intracranial Space Occupying Lesion, Post Encephalitic, Hypoxic Encephalitic, Chronic Subdural Haematoma and Tubercular Meningitis. Conclusion: Vascular dementia is more than Alzheimer's Dementia in Bangladesh. Multiple vascular risk factors contribute to this.
\end{abstract}

Key words: Dementia, Alzheimer's disease, elderly people

\section{Introduction}

Dementia is a syndrome due to disease of the brain - usually of a chronic or progressive in nature in which there are disturbances of multiple higher cortical functions, including memory, thinking, orientation, comprehension, calculation, learning capacity, language, and judgment. Consciousness is not clouded ${ }^{1,2}$. The impairments of cognitive function are commonly accompanied, and occasionally preceded, by deterioration in emotional control, social behavior, or motivation ${ }^{2}$. Dementia is typically diagnosed when acquired cognitive impairment has become severe enough to compromise social and/or occupational functioning. ${ }^{2,3}$ Mild cognitive impairment $(\mathrm{MCl})$ is a state intermediate between normal cognition and dementia, with essentially preserved functional abilities $^{1-4}$.

Dementia mainly affects older people, more than 65 year of age. The prevalence doubles with every five-year increment in age after 65 There is a fourfold variation in prevalence overall, from $2.07 \%$ in West Sub-Saharan Africa to $8.50 \%$ in Latin America among persons aged $>60$ years ${ }^{5,6}$. However, most of the estimated age-standardized prevalence figures lie in a band between $5 \%$ and $7 \%{ }^{6}$. The prevalence is estimated to be $6.38 \%$ in South East Asia ${ }^{6}$. Life expectancy is increasing across the world, with population aging increasing the most rapidly in low-income and middle-income countries, where the prevalence of dementia is therefore expected to increase ${ }^{5}$.

Dementia occurs in a large number of conditions primarily or secondarily affecting the brain. Alzheimer's disease is the most common form of dementia, irreversible and possibly contributes to $60-70 \%$ of cases $^{2,7}$ Vascular dementia, either in the form of multi-infarct dementia or post-stroke dementia, is the second most common cause of dementia, accounting for as many as $40 \%$ of

1. Professor, Department of Neurology, BSMMU, Dhaka

2. Associate Professor, Department of Neurology, BSMMU, Dhaka

3. Associate Professor, Department of Gastroenterology, BSMMU,Dhaka

4. Medical Officer, Department of Neurology, BSMMU,Dhaka

5. Medical Officer, Cantonment General Hospital ,Dhaka

6. Medical Officer, Fast Care Hospital,Dhaka 
cases $^{2-4}$. Dementia may develop late in Parkinson's disease ${ }^{4}$. Dementia with Lewy bodies, and a group of diseases that contribute to fronto-temporal dementia are important but rare causes. The boundaries between subtypes are indistinct and mixed forms often co-exist ${ }^{2}$. Infections of brain structures, such as meningitis and encephalitis due to tuberculosis or other infections, such as HIVI AIDS and syphilis, can affect the brain permanently in later stages and can be primary causes of dementia ${ }^{2-6}$. Chronic Subdural Haematoma or other causes of intracranial space occupying lesion may lead to dementia ${ }^{5}$. Sudden, severe hypoxia may also cause brain damage and symptoms of dementia ${ }^{2.4-7}$. Dementia in people with chronic alcoholism is believed to result from other complications such as liver disease and nutritional deficiencies ${ }^{2.4-7}$.

Age may be a risk factor in itself or may reflect the effect of increasing time during which other factors can exert their influence. Cardiovascular risk factors are smoking, high blood pressure, diabetes and hyperlipidaemia ${ }^{8}$. All of these are independent risk factors for the development of vascular dementia and predispose to the development of atherosclerosis, which is associated with dementia of all clinical types. These risk factors also predispose to acute stroke, which is well established as a risk factor for the development of dementia ${ }^{8,9}$. Current literature suggests that there is a causal relation between vascular mechanisms and the development of non-genetic AD. Smoking, high blood pressure and diabetes mellitus are all independent risk factors for the development of $A D^{10-12}$. Life expectancy in Bangladesh in 2012 was 69 years for male and 71 years for female. Above 65 years population is 7.6 million $(4.8 \%$ of total population) ${ }^{13}$.

Number of population in older age group is increasing. So, patients of neurodegenerative disorder including dementia is increasing. Because all types of dementia are treatable, at least with psychosocial interventions, accurate diagnosis and evaluation of etiological pattern is essential to determine the appropriate treatment and to provide information about prognosis, possible genetic risks, and health care planning to the patient and family.
With this intention this study was carried out to explore the etiological pattern of dementia in a tertiary care hospital.

\section{Methods:}

This cross sectional study was carried out in the department of Neurology, Bangabandu Sheikh Mujib Medical University, Dhaka, Bangladesh from January 2012 to December 2012. Consecutive 106 patients of any age with history suggestive of dementia from the Neurology outpatient department of BSMMU were initially enrolled.

The history and findings of general and neurological examinations were recorded in a semi-structured data sheet. Neuropsychological assessments were done by mini mental state examination (MMSE) $)^{1,14}$ and 88 patients with MMSE score 24 or less were diagnosed as dementia. Patients complaining of loss of memory but score obtaining 25 or more were diagnosed as minimal cognitive impairment $(\mathrm{MCl})$ and were excluded from the study. Patients with post-traumatic amnesic state and acute confusional state were also excluded.

Neuro-imaging, either computed tomography (CT) or magnetic resonance imaging (MR1) was done for all patients. Laboratory tests including complete blood count, blood sugar, serum creatinine, serum electrolytes, liver function tests, thyroid function tests and serum vitamin B12 assay were also done for all patients. Venereal diseases research laboratory (VDRL) test was done in selected cases. Alzheimer's disease and vascular dementia (VD) were diagnosed on the basis of DSM-IV criteria ${ }^{1,15}$.

Written informed consent was obtained from each patient. The study protocol was approved by the ethical committee and institutional review board (IRB) of BSMMU.

\section{Results:}

Among 88 patients diagnosed to have dementia, $57(65 \%)$ were male and $31(35 \%)$ were female. Age range was 32-85 years with mean age of 67.9 . Most of the patients $(40,45.5 \%)$ belonged to 70 74 years age group followed by $65-69(24,27.2 \%)$ years age group.

Among the demented patients, 38 (43.2\%) were retired from their profession. Housewives $(29,33 \%)$ 
were also of significant number. Most (35, $33.7 \%)$ were illiterate and only $14(13.5 \%)$ completed graduation. (Table-I \& Table-II)

Table I

Socio-demographical status of the patients

\begin{tabular}{lcc}
\hline & $\mathrm{n}$ & $\%$ \\
\hline Gender $(\mathrm{n}=88)$ & & \\
Male & 54 & 70.2 \\
Female & 34 & 29.8 \\
Education $(\mathrm{n}=88)$ & & \\
Illiterate & 29 & 33.7 \\
Primary & 33 & 30.8 \\
Secondary & 14 & 19.2 \\
HigherSecondary & 6 & 2.9 \\
Graduate andAbove & 6 & 13.5 \\
Marital status (n=88) & & \\
Married & 73 & 86.0 \\
Single & 1 & 1.0 \\
Widow & 14 & 13.0 \\
Occupation $(\mathrm{n}=88)$ & & \\
Day laborer & 5 & 5.7 \\
Service & 9 & 10.2 \\
Housewife & 29 & 33.0 \\
Business & 2 & 2.3 \\
Retired & 38 & 43.2 \\
Farmer & 5 & 5.7 \\
\hline
\end{tabular}

Table-II

Age distribution of demented patient

\begin{tabular}{lc}
\hline Age & Number of patient \\
\hline $30-49$ & $4(4.54 \%)$ \\
$50-54$ & $4(4.54 \%)$ \\
$55-59$ & $7(7.95 \%)$ \\
$60-64$ & $13(14.77 \%)$ \\
$65-69$ & $14(15.90 \%)$ \\
$70-74$ & $20(22.72 \%)$ \\
$75-79$ & $6(6.81 \%)$ \\
$80-84$ & $13(14.77 \%)$ \\
$85-89$ & $7(7.95 \%)$ \\
\hline
\end{tabular}

In our study 52 patients had history of stroke, 43 patients were hypertensive, 21 patients were diabetic, 8 patients were suffering from ischemic heart disease and 5 patients were suffering from dyslipidaemia. (Table-III)
Table-III

Distribution of patients according to diseases accompanying dementia

\begin{tabular}{lc}
\hline $\begin{array}{l}\text { Diseases accompanying } \\
\text { dementia }\end{array}$ & Number ofpatients \\
\hline DM & 21 \\
HTN & 43 \\
Dyslipidaemia & 5 \\
IHD & 8 \\
Stroke & 52 \\
\hline
\end{tabular}

Vascular dementia was the underlying diagnosis in most of the case i.e. 45 patients(43.3\%) followed by Alzheimers Disease $(21,20.2 \%)$ and Parkinsons Disease $(8.7,9 \%)$. Other causes were Mixed Dementia (4), Intracranial space occupying Iesion(3), Post Encephalitic(1), Hypoxic encephalitic (1), Chronic Subdural Haematoma (1) and Tubercular Meningitis(1). (Table-IV)

Table-IV

Distribution of dementia patients by underlying diseases

\begin{tabular}{lcc}
\hline Diagnosis & Frequency & Percentage \\
\hline Alzheimers Disease & 21 & 20.2 \\
Vuscular Dementia & 45 & 43.3 \\
Mixed Dementia & 4 & 3.8 \\
Parkinsons disease & 9 & 8.7 \\
Post Encephalitic & 1 & 1.0 \\
ICSOL & 3 & 2.9 \\
Hypoxic encephalitic & 1 & 1.0 \\
Chronic Subdural Haematoma & 1 & 1.0 \\
Tubercular Meningitis & 1 & 1.0 \\
\hline
\end{tabular}

Discussion:

Male were predominant (65\%) in this study. Ratio between male and female was 1.8:1. A similar study carried out in BSMMU by Islam MN et al was also dominated by male $(62.4 \%)^{16}$. Hoffman et al ${ }^{17}$ found that the prevalence of dementia was slightly higher in men then in women among subjects under 75 years. On the contrary, Kukull et al reported that sex did not affect dementia, which is not consistent with the present study although the occurrence of non-AD dementia was found significantly less frequent in women than in men $^{18}$. In the present 
study, cases were enrolled from Neurology department of BSMMU, both inpatient and outpatient, where most of the patients were male. In Bangladesh, due to socio-cultural practice, female patients are less likely to seek medical help for their illness than male. This may be the reason of the inconsistency of the distribution of sex in this study.

In this study, most of the patients (45.5\%) belonged to $70-74$ years age group and most were retired from their profession. Dementia mainly affects older people, more than 65 year of age. The prevalence doubles with every five-year increment in age after 65.6 McDowell et $\mathrm{al}^{19}$ and Nunes $\mathrm{B}$ et al ${ }^{20}$ showed that prevalence of dementia was increasing steeply with age.

Most of the patients $(33.7 \%)$ in this study were illiterate and only $13.5 \%$ completed graduation. Qiu C et al, 2001 observed in the Kungsholmen Project that, the association between a low level of education ( $<8$ years) and an increased incidence of $A D$ or dementia was present independently of age, sex, baseline cognitive performance, vascular disease, and socioeconomic status ${ }^{21}$. Letenneur $L$ et al also found low educational attainment to be associated with a higher risk of Alzheimer's disease ${ }^{22}$. Kukull WA et al stated that a higher educational level is associated with decreased risk of both $A D$ and non-AD dementia, or, conversely, a lower educational level is associated with an increased risk. Subjects who have more than 15 years of education were at nearly half the risk of subjects with less than 12 years of education. ${ }^{18}$ Similar result was reported by Monorey JT et al ${ }^{8}$.

The distribution of major categories of dementia was recorded in this study. Vascular dementia was found the highest number (43\%), followed by Alzheimer's disease (20\%), Parkinson's disease $(8.7 \%)$, mixed dementia (3.8\%). In developed countries, Alzheimer's disease is the main cause of dementia. Alzheimer's Research Trust states that in England, Alzheimer's disease is the most common cause of dementia, accounting for about $60 \%$ of all cases, followed by vascular dementia and dementia with Lewy bodies which together account for $15-20 \%$ cases $^{23-25}$. Suh et al mentioned that Alzheimer's disease (AD) has become nearly twice as prevalent as vascular dementia (VaD) in Korea, Japan, and China since transition in early 1990s. Prior to this, in the 1980s, $\mathrm{VaD}$ was more prevalent than $A D$ in these countries. In Nigeria, the prevalence of dementia was low. Indian studies were contradictory, with both $\mathrm{AD}$ and $\mathrm{VaD}$ being more prevalent in different studies.

Stroke, HTN, DM, IHD and Dyslipidaemia (52, 43, 21,8 and 5 patients respectively) were the diseases accompanying dementia in this study. There were frequent overlap e.g. same patient were suffering from stroke, DM and Dyslipidaemia. HTN, DM, IHD and Dyslipidaemia are recognized risk factor of stroke and stroke may lead to vascular dementia which was found to be $43.3 \%$ in this study.

\section{Conclusion:}

In Bangladesh, Vascular dementia is more than Alzheimer's Dementia. Multiple vascular risk factors contribute to this. Patients should have regular cardiovascular risk factors screening to prevent this dementia.

Limitations of study. Prevalence of dementia could not be known as only demented patients from a tertiary care hospital were included. Community and population-based studies are required to obtain accurate epidemiological data on dementia in Bangladesh. The diagnostic criteria used to diagnose and differentiate dementia subtypes have less than ideal sensitivity and specificity and syndromes overlaps \& multiple pathologies could be present. Moreover validation and adaptation of many western neuro-cognitive, behavioral, functional scales and questionnaires are required for diagnosis of dementia in our socio-cultural perspective.

\section{Acknowledgement:}

The authors express their gratitude to the Centre for Excellence Development Project of Bangabandhu Sheikh Mujib Medical University, Bangladesh for funding the project.

\section{References:}

1. The ICD-10 classification of mental and behavioural disorders. Clinical descriptions 
and diagnostic guidelines. Geneva, World Health Organization, 1992.

2. Dekosky ST, KauferDI, Hamilton RL, Wolk DA, Lopez OL. Dementia. In: Bradley WG, daroffRB, Fenichel GM, Jancovic J (eds). Neurology in clinical practice: $5^{\text {th }}$ ed. Philadelphia: Elsevier; 2008. p.1856.

3. Bird ID, Miller BL. Dementia. In :Fauci AS, Kasper DL, Longo DL, Braunwald E, Hauser SL, Jameson JL. Harrisons's principles of internal medicine. $17^{\text {th }}$ ed. Newyork : McGraw-Hill; 2008. p. 2536

4. Hugo J, Ganguli M. Dementia and Cognitive Impairment Epidemiology, Diagnosis and Treatment. Clin Geriatr Med. 2014; 30 : 42142.

5. Prince $M$, Prina M, Guerchet M. World Alzheimer report 2013. Journey of caring: An analysis of long term care for dementia. Alzheimer's Disease International. Available at: http://www. alz.co. ukiresearch/worldreport-2013.

6. Prince M, Bryce R, Albanese E, Wimo A, Ribeiro W, Ferri CP. The global prevalence of dementia: A systematic review and metaanalysis. Alzheimer's \&Dementia. 2013; 9: 63-75

7. Shelley B P, Khabouri J A. The Spectrum of Dementia : Frequency, Causes and Clinical Profile. Dement Geriatr Cogn Disord. 2007; 24:280-287.

8. Monorey JT, Tang MS, BergkindL, Scott M, Merchant C, Bell K, Stern Y, Mayeux R. Low density lipoprotein cholesterol and the risk factor of dementia with stroke. JAMA. 1999; 282(3):254-260.

9. Catriona D, McCullagh, Craig D, Stephen P, Passmore PP. Risk factors of dementia. Advances in Psychiatry Treatment. 2001; 7 : $24-31$.

10. Masellis M, Sherborn $K$, Neto PR, Sadovnick DA, Hsiung GR, Black SE. Early-onset dementias: diagnostic and etiological Considerations. Alzheimer's Research \&
Therapy. 2013; 5 (Suppl 1):S7.

11. Makin. SDJ, Turpin S, Dennis MS, Wardlaw JM. Cognitive impairment after lacunar stroke: systematic review and meta-analysis of incidence, prevalence and comparison with other stroke subtypes. J Neurol Neurosurg Psychiatry. 2013; 84: 893-90.

12. De la Tone JC. Is Alzheimer's disease a neuro-degenerative or a vascular disorder? Data, dogma, and dialectics. Lancet Neurol. 2004; 3: 184-190.

13. Health statistics of Bangladesh. World health organization, 2012. Available from: www.who.int/countries/bgdien.

14. Folstein, M., Folstein, S. and McHughs, P. The Mini-mental state: a practical method for grading the cognitive state of patients for the clinician. Journal of Psychiatric Research.1975; 12:189-98.

15. American Psychiatric Association. Diagnostic and statistical manual of mental disorders, 4thed (DSM-IV). Washington DC: American Psychiatric Association, 1994.

16. Islam MN, Khan RK, Rahman MM, Hayee MA, Jahan ME, Bhuiya MM. Etiological pattern of demented patients attending a tertiary care hospital. Mymensingh Med J. $2013 \mathrm{Jul} ; 2$ 22(3):496-503.

17. Hofman A, Rocca WA, Brayne C, BretelerMmb, Clarke M, Cooper B. International Journal of Epidemiology. The Prevalence of Dementia in Europe: A Collaborative Study of 1980-1990 Findings. 1991;20(3):736-48

18. Kulull WA, Higdon R, Bowen JD, McCormick WC, Teri L, Schellenberg GD. Dementia and Alzheimer disease incidence: a prospective cohort study. Arch Neurology. 200; 59(11):1737-46.

19. McDowell I. Canadian study of health and aging : study methods and prevalence of dementia. CMAJ. 1994;150(6):899-9

20. Nunes B, Silva RD, Cruz VT, Roriz JM, Pais $J$, Silva M. Prevalence and pattern of cognitive 
impairment in rural and urban population from Northern Portugal. BMC Neurology 2010;10:42

21. Qiu C, Backman L, Winblad B, Agitero-Torres $H$, Fratiglioni $L$. The influence of education on clinically diagnosed dementia incidence and mortality data from the. Arch Neurol. 2001 Dec;58(12):2034-9.

22. Letenneur L, Gilleron V, Commenges D, Helmer C, Orgogozo JM, Dartigues JF. Are sex and educational level independent predictors of dementia and Alzheimer's disease? Incidence data from the PAQUID project. J NeurolNeurosurg Psychiatry. 1999 Feb;66(2):177-83.
23. Luengo-Fernandez R, Leal J, Gray A. Dementia 2010. Health Economics Research Centre, University of Oxford for the Alzheimer's Research Trust. Available from : www.dementia2010.org

24. Ravaglia G, FortiP, Maioli F, Martelli M, Servadei L, Brunetti N. Incidence and etiology of dementia in a large elderly Italian population. Neurology. 2005:64(9):1525-30

25. Prencipe M, Casini AR, Ferretti C, Lattanzio MT, Fiorelli M, Culasso F. Prevalence of dementia in an elderly rural population: effects of age, sex and education. J Neurol Neurosurg Psychiatry. 1996; 60(6):628-33. 